\title{
Modelo de Simulación de un Sistema Logístico de Distribución como Plataforma Virtual para el Aprendizaje Basado en Problemas
}

\author{
Edgar L. Giraldo-Picon ${ }^{1}$, Jaime A. Giraldo-García ${ }^{1}$ y Jorge A. Valderrama-Ortega ${ }^{1}$ \\ (1) Universidad Nacional de Colombia, Facultad de Ingeniería y Arquitectura, Departamento de Ingeniería \\ Industrial, GTA-IDT, Campus La Nubia Bloque Q piso 2, Manizales - Colombia. \\ (e-mail: elgiraldop@unal.edu.co; jaiagiraldog@unal.edu.co; javalderramao@unal.edu.co)
}

Recibido Feb. 28, 2018; Aceptado May. 14, 2018; Versión final Jun. 22, 2018, Publicado Dic. 2018

\begin{abstract}
Resumen
Se ha desarrollado una plataforma virtual de simulación de sistemas de producción industrial con el objetivo de analizar las operaciones de un sistema logístico de distribución de pequeña escala, dirigida especialmente a estudiantes y profesionales involucrados en la planeación, diseño y operación de los sistemas logísticos. La plataforma está basada en la metodología del Aprendizaje Basado en Problemas, la cual presenta un conjunto de problemas construidos por grupos de profesores o profesionales. Estos problemas requieren ser investigados por el usuario para discutir, producir explicaciones y proponer soluciones fundamentadas. Los resultados de los escenarios simulados demuestran como el cambio de una variable afecta el desempeño del sistema y que otros factores se deben considerar para realizar una evaluación más amplia y concreta. Basado en los resultados del estudio, la herramienta permite a los estudiantes y profesionales experimentar creando escenarios y analizar diferentes situaciones.
\end{abstract}

\section{Simulation Model of a Logistic Distribution System as a Virtual Platform for Problem-Based Learning}

\begin{abstract}
A virtual simulation platform for industrial production systems has been developed with the objective of analyzing the operations of a small-scale distribution logistics system, aimed especially at students and professionals involved in the planning, design and operation of logistics systems. The platform is based on the methodology of problem-based learning, which presents a set of problems proposed by groups of teachers or professionals. These problems require to be investigated by the user to discuss, produce explanations and propose founded solutions. The results of the simulated scenarios show how the change of a variable affects the performance of the system and that other factors must be considered in order to carry out a broader and more concrete evaluation. Based on the results of the study, the tool allows students and professionals to experience creating scenarios and analyzing different situations.
\end{abstract}

Keywords: distribution logistics systems; simulation; $A B P$; industrial training 
Modelo de Simulación de un Sistema Logístico de Distribución como Plataforma Virtual $\quad$ Giraldo-Picon

\section{INTRODUCCIÓN}

La dinámica empresarial que se desarrolla en el mundo globalizado, requiere de la adopción y aplicación de competencias profesionales que respondan de forma oportuna y asertiva a los rápidos procesos de cambio en las organizaciones. Según Blanco (2010), Empresa, Innovación y Desarrollo constituyen un acercamiento a la empresa como nodo de interacciones, no sólo desde la concepción de unidad económica de producción sino como nodo de decisiones de agentes. En este sentido, es necesario que la formación de aquellos individuos que estarán involucrados en los procesos de toma de decisiones, esté orientada de forma apropiada y alineada con la realidad empresarial. Si bien, la oferta académica y empresarial es cada vez mayor, es evidente que los procesos de enseñanza-aprendizaje no han sufrido una transformación reconocible en función de las necesidades que actualmente requiere el mundo globalizado, las cuales crecen a un ritmo acelerado y son cada vez más especializadas. Soca (2015) expresa que se deben constituir elementos de importancia para apoyar no solo el proceso de aprendizaje del área a tratar sino la creación de la capacidad de aprender a aprender.

Es de considerar que conforme avanza el tiempo, a las compañías se les exige más en términos de productividad, competencias y calidad en los profesionales que llevan consigo grandes responsabilidades, por ejemplo, toma de decisiones (Villegas, 1996). Sin embargo, adquirir este nivel en los profesionales es una tarea compleja debido a la falta de experiencia, formación académica débil y/o falta de motivación. Por esta razón, las compañías crean programas de capacitación y entrenamiento del personal para tareas específicas en donde permitan al usuario experimentar situaciones o escenarios que ayuden al desarrollo del mismo, teniendo en cuenta que el entrenamiento del personal se considera un medio de desarrollo de competencias en las personas para que sean más productivas, creativas e innovadoras, y puedan contribuir mejor a los objetivos organizacionales (Chiavenato, 2002).

Las personas tienen una increíble capacidad para aprender y para desarrollarse, y la educación está en el centro de esa capacidad. Los procesos de desarrollo de las personas tienen una estrecha relación con la educación (Chiavenato, 2009). De igual forma, el qué y el cómo se aprende y el qué y cómo se enseña continúa siendo en buena medida terreno intocado. Este cuestionamiento se ha evitado tanto en la pedagogía como en el análisis sociológico del aparato escolar y empresarial. En verdad el tema del saber, ese conjunto de recursos simbólicos que incluyen dimensiones tanto de conocimientos como de valores, habilidades instrumentales y disposiciones, debería estar en el centro de la problemática de la calidad y de la preocupación política por la Educación Superior (Parra, 2006).

En este sentido, y teniendo en cuenta que no es posible pretender que se produzca una transformación completa de los métodos de enseñanza tradicionales empleados de forma extensa en la mayoría de los escenarios educativos y empresariales, deben generarse mecanismos que permitan una mejor articulación con las demandas del entorno y que a su vez aporten de forma paulatina a una reestructuración del sistema. Según Parra (2006), la diferencia fundamental entre el método de enseñanza tradicional y el moderno se centra en que, en el primero, el proceso de enseñanza-aprendizaje es estático, lineal y rígido, mientras en el segundo, el mismo proceso implica una continua retroalimentación, es abierto y dúctil y sus componentes adquieren significado en el contexto sociocultural en el que la persona está inmersa. La adopción de nuevos enfoques implica que la información transmitida sea relevante para la persona, reconociendo su utilidad y aplicabilidad hacia sus necesidades particulares y su relación con el entorno empresarial.

El proceso de aprendizaje, debe estar de frente a los retos del avance vertiginoso y diversificado de la ciencia y las tecnologías y su aplicabilidad a través de la práctica de la profesión. Por ello el conocimiento y el ejercicio de nuevas experiencias en los procesos de formación, son tan importantes (ACOFI, 2008). Al referirse a nuevas experiencias se habla de aquellas estrategias que permiten al usuario construir su perfil profesional a partir de la experiencia práctica, propiciando el pensamiento crítico-reflexivo bajo la confrontación de situaciones que se asemejen a problemas reales de su profesión. Atendiendo a lo anterior, durante los últimos años, ha surgido una tendencia enfocada en el desarrollo de metodologías educativas centradas en el aprendizaje de la persona, dentro de las cuales se encuentra el Aprendizaje Basado en Problemas (ABP), una metodología que reconoce al estudiante y al profesional como actores fundamentales y responsables de su propio proceso de aprendizaje. Valderrama y Castaño (2017) explican que el ABP promueve en el estudiante el aprendizaje activo y significativo, que le permite solucionar problemas reales de conocimiento en torno a una temática o área específica, además de recolectar y procesar información de manera eficiente a partir de la tutoría funcional del profesor. El ABP presenta un conjunto de problemas construidos de forma estructurada por grupos de profesionales, los cuales requieren de investigación por parte del usuario (estudiante) para discutir, producir explicaciones tentativas de la problemática y proponer soluciones fundamentadas (Norman y Schmidt, 1992). De igual manera, Tortorella y Cauchick (2017) y Rakhudul et al. (2017) coinciden que en los cursos de ABP los estudiantes trabajan en equipo para resolver problemas complejos y auténticos que permiten desarrollar el conocimiento del contenido y la resolución de problemas, 
el razonamiento, la comunicación y las habilidades de autoevaluación. Esto, teniendo en cuenta que en las últimas décadas se ha notado un creciente interés en utilizar ABP en la educación de ingeniería, que está alineado con los esfuerzos actuales para pasar de la presentación descontextualizada de contenido técnico a la integración holística de contenido y práctica (Sheppard et al., 2008).

Ahora bien, desde el enfoque de los sistemas logísticos, ha de reconocerse que la toma de decisiones de forma asertiva en torno a la planeación, el diseño y la operación de los mismos, por parte de los profesionales de ingeniería y administración, posee un nivel de importancia crítico y estratégico, ya que genera un impacto directo sobre el desempeño de las compañías. Marwala (2013 - A) explica que la toma racional de decisiones es un proceso a través del cual se toma una decisión utilizando cierta información recopilada e inteligencia para tomar una decisión optimizada. De igual forma, Marwala (2013 - B) presenta que la toma de decisiones racional puede definirse como el proceso a través del cual se toma una decisión, utilizando un enfoque sistemático, para resolver un problema de una manera lógica, práctica y objetiva. Cada sistema, por su naturaleza, presenta características diferentes e involucra un número de variables extenso, de las cuales, cada una puede afectar de forma importante el comportamiento del mismo y cuya aleatoriedad e interdependencia genera un número de escenarios y situaciones ilimitadas. Elegir una solución precisa para una problemática que se presente, no resulta sencillo y dependiendo de la envergadura del sistema, evaluar el impacto de la misma puede ser inaccesible debido a las implicaciones de costos que representa la alteración del funcionamiento normal del sistema.

Por otro lado, se resalta que el recurso humano actual de las empresas desarrolla competencias a través de la experiencia empírica en el trabajo y muchas veces son obtenidas de cursos externos basados en problemáticas de casos estándares. Sin embargo, la dependencia del saber-hacer de las compañías en cursos de capacitación externos de su recurso humano falla en el proceso en el cual el usuario debe transferir sus conocimientos y habilidades de la capacitación o curso externo al trabajo real de la compañía. Según Villegas (1996) para enfrentar estas dificultades se deben dominar los problemas teniendo en cuenta tres áreas principales: 1) procesos de entrenamiento industrial, 2) aprendizaje organizacional y 3) creación de entornos virtuales de experimentación mediante simulación.

El entrenamiento industrial consiste en ayudar a las personas a aprender a trabajar de manera más efectiva y reconocer su relevancia en toda la industria (Villegas, 1996). De esta manera, la persona logra desarrollar las competencias necesarias para la realización de una actividad, considerando que las competencias son el producto de una experiencia buscada y explotada activamente por aquel que participa en ella, experiencia que permite la integración con éxito de los conocimientos a fin de construir competencias inéditas (Alles, 2007). Las empresas necesitan contar cada vez más con nuevas competencias, por lo tanto, la capacidad de aprender es cada día más requerida. Este planteamiento considera que las compañías deben enfocarse en la realización y aplicación de métodos y/o herramientas que abarquen y propongan gran cantidad de aspectos evaluables para el desarrollo de las capacidades de los profesionales involucrados en ambientes logísticos. Motsoeneng et al. (2015) mencionan que el objetivo del entrenamiento es alterar el comportamiento de las personas y que el aprendizaje se lleva a cabo principalmente en el trabajo, especialmente en el caso de los ingenieros, que a menudo adquieren experiencia a través de la capacitación práctica. Además, es muy importante que el desarrollo de los ingenieros les ayude a hacer frente a los continuos cambios tecnológicos y las innovaciones en su industria. Aunque estos autores mencionen solo a los ingenieros, la definición realiza un contraste general para aquellos involucrados en sistemas logísticos. Por ejemplo, Caicedo, Niño y Romero (2013) destacan la falta de preparación de los gerentes de producción actuales, en donde estos deben estar mejor versados en diversas tecnologías y mostrarse capaces de manejar sistemas de información y soporte complejos.

Sin embargo, la selección y aplicación de técnicas de capacitación es un tema que para las compañías sigue siendo complejo, esto a causa de la deficiencia del recurso humano en la transferencia del conocimiento a la compañía debido a que en la mayoría de los cursos de capacitación enseñan a las personas acerca de lo que no se conoce, mientras que debería enseñarse sobre lo que ya se conoce (Villegas, 1996, p. 39). Por lo tanto, el uso de sistemas simulados acerca al usuario con la compañía y transfiere el conocimiento de forma eficiente, ya que su fundamento es que el usuario adquiera los conocimientos prácticos necesarios en condiciones seguras, sin riesgo, con las ventajas de una disminución del estrés y la capacidad de tolerar y corregir los errores de rendimiento (Vanyolos et al, 2017).

Por otra parte, el aprendizaje organizacional, es un tema donde diferentes disciplinas y teóricos debaten debido a su amplio campo y complejidad. López, et al. (2017) exponen que el conocimiento organizacional es considerado como un factor de la producción igual o más importante que la tierra, el trabajo y el capital, y como una fuente de energía posmoderna, a la altura de la electricidad o el petróleo en su época. Garzón y Fisher (2008) expresan que el aprendizaje organizacional es un fenómeno compuesto y complejo, con investigaciones desarrolladas dispares sobre este campo y sus resultados poco acumulativos. Monti, et al. 
(2016) explica que para lograr aprendizaje organizacional es necesario desarrollar mecanismos de captación, almacenamiento, interpretación, transmisión, producción y evaluación de información y conocimiento, que permitan aprovechar al máximo el aprendizaje que se da a nivel de las personas y de los equipos de proyectos, donde estos últimos superan el desempeño individual cuando las tareas que se desarrollan requieren de habilidades múltiples, sentido común y experiencia. Sin embargo, este proceso de aprendizaje puede fallar. Villegas (1996) expone tres razones básicas del porque falla el aprendizaje organizacional: aprendizaje situacional, donde se desarrolla la solución de un problema, pero no se lleva el aprendizaje de éste al próximo caso; aprendizaje fragmentado, donde el individuo aprende, pero no la organización; y, el aprendizaje oportunista, donde las acciones de la organización se toman sobre la base de una acción individual y no en los modelos ampliamente compartido de las organizaciones. Por lo tanto, se debe considerar que en la realización de estos procesos generalmente se genera un proceso de aprendizaje individual en el cual solo una persona es la que desarrolla las habilidades para la solución de problemas, condicionando así a la compañía a una sola persona. Estas situaciones se presentan de forma constante en las empresas actualmente, en donde la salida inesperada del individuo con el conjunto o marco de habilidades para el funcionamiento de la empresa, arriesgarían su estabilidad y permanencia en el mercado. Conceptos como es imperativo retener a los empleados clave que ocupan puestos que son críticos para el éxito de la organización (Koekemoer, 2014) difieren del concepto de aprendizaje organizacional.

Sin embargo, esto se puede solucionar conociendo las habilidades y conocimientos que tiene y debería tener el recurso humano en las compañías, para así, explotar las existentes y desarrollar las faltantes de manera equilibrada entre éstos. Según Saravanan (2006) las habilidades de comunicación, pensamiento crítico y resolución de problemas, trabajo en equipo, el aprendizaje permanente, gestión de la información, emprendimiento, ética y habilidades morales y profesionales, y liderazgo son esenciales para aquellos ingenieros o personas que se desempeñen en ambientes logísticos. De igual manera, Seat y Lord (1999) mencionan que las entrevistas, el interrogatorio, el intercambio de ideas y el manejo de conflictos también son habilidades de ingeniería vitales; y Bodmer et al. 2002 explica que muchos involucrados en este campo presentan lagunas de competencia en el área de comunicación, gestión del tiempo, resolución de problemas, aplicación de conocimientos en el lugar trabajo, capacidad para hacer frente a la presión y capacidad para aprender. Debe ser de vital importancia para las organizaciones empresariales e institucionales desarrollar (en su mayoría) estas habilidades. Esto, para obtener un mejor recurso humano, generar un buen aprendizaje organizacional y así afrontar rápidamente los constantes cambios de este mundo globalizado. Dicho esto, en tal variedad de competencias básicas se puede encontrar la razón principal que ha llevado a los practicantes estudiantes y profesionales a postular que ninguna técnica de capacitación independiente puede hacer frente a todos estos requisitos y que los mejores resultados se pueden lograr cuando los proyectos de capacitación son el resultado de una combinación de los métodos tradicionales de aprendizaje, como el aprendizaje instructivo con otras técnicas de aprendizaje más avanzadas y orientadas a la práctica, como la simulación por computadora (Villegas, 1996).

El área de simulación basada en computadoras es una de las herramientas que se ha empleado de forma extensa con la utilización de las Tecnologías de la información y la comunicación (TIC). Ésta es una técnica que permite imitar o simular la operación de diferentes clases de instalaciones o procesos del mundo real o hipotético haciendo uso de las computadoras (Giraldo, 2014), con lo cual es posible obtener información clave del sistema en diferentes escenarios, sin necesidad de perturbarlo. Con las herramientas de simulación se pueden desarrollar modelos robustos que se acercan a la realidad estudiada, cuestión que no pasa con otras herramientas de soporte a la toma de decisiones, tales como: la programación lineal o algunos métodos de solución analítica como el cálculo (diferencial e integral) y el álgebra, entre otros (Kelton y Law, 2007). Por otro lado, Vázquez et al. (2009) y Fonseca et al. (2016) coinciden en que los ambientes simulados virtuales ofrecen soporte para el desarrollo del pensamiento crítico, resolución de problemas, flexibilidad y adaptabilidad a los estudiantes y profesionales que se involucren o estén involucrados en esta área.

De esta manera, el uso de simulación está relacionado con la complejidad de los sistemas estudiados, haciendo intervenir variables controlables y no controlables, con características estocásticas y alta interdependencia entre componentes, mientras que con el uso de otras herramientas matemáticas se deben proponer algunas hipótesis de simplificación, las cuales alejan el sistema estudiado de su realidad (Vargas y Giraldo, 2015). Partiendo de esto, es posible reducir el riesgo al tomar decisiones fundamentadas en la experimentación, anticipando en alguna medida los efectos que éstas tendrían sobre el desempeño del sistema real. Las aplicaciones desarrolladas para este objetivo son variadas y en muchas ocasiones orientadas al análisis de sistemas o áreas de estudio específicas. Partiendo de los planteamientos anteriores se ha elaborado un modelo de simulación por eventos discretos, basado en el proceso de fabricación y distribución de productos tangibles realizados por una compañía para 10 clientes ubicados en diferentes ciudades. La plataforma permite modificar diferentes variables de decisión que afectan el funcionamiento del sistema a través de una interfaz de Microsoft Excel, entre las cuales se incluyen el tiempo entre pedidos, número de unidades requeridas por cliente, tiempo de procesamiento, tiempo de cargue y descargue, 
velocidad de los vehículos, entre otros. En base a estos, se pueden obtener diferentes resultados para diferentes escenarios y a partir de ello determinar cambios que son necesarios para mejorar el desempeño del sistema estudiado. Se espera que con esta herramienta tanto estudiantes, profesionales y tutores experimenten y analicen diferentes situaciones problemáticas, con el fin de fortalecer los conocimientos de los mismos acerca de los sistemas logísticos y las habilidades para la solución de problemas acorde con la metodología $A B P$, contribuyendo al mejoramiento de los procesos de enseñanza aprendizaje en las instituciones y empresas que se emplean en la actualidad.

\section{METODOLOGÍA}

Para el desarrollo de la plataforma de simulación del sistema de distribución logístico a pequeña escala se ha tomado como base la metodología propuesta por Giraldo (2014), la cual es una adaptación propia a partir de los aportes de una serie de autores de gran relevancia en el ámbito de la simulación cómo Kelton y Law (2007) y cuyo contenido describe las etapas que conforman el procedimiento de la planeación y experimentación de un modelo de simulación.

\section{Formulación del problema}

Siguiendo el enfoque planteado para el modelo como herramienta de enseñanza-aprendizaje basada en la metodología ABP, la formulación de un problema específico no es delimitada de forma estricta, ya que estará determinada en función del mismo o bien del caso de estudio planteado por el usuario o tutor que decida implementar la herramienta. Sin embargo, para el desarrollo del aplicativo se parte de la situación actual de la empresa de interés y a partir de ésta se analizan diversas problemáticas según la lógica causa-efecto alrededor de la logística de distribución que pueden originar la problemática identificada y que podrían ser evaluadas mediante la utilización de la plataforma desarrollada.

La situación actual enfrentada es la siguiente: La empresa presenta problemas para atender las órdenes de pedido de los clientes de forma oportuna. Esta situación ha generado diversos inconvenientes con los clientes, generando una reducción en la frecuencia en los pedidos, con lo cual disminuyen los ingresos de la organización y se ve considerablemente afectada la percepción que se tiene de la empresa en la región.

Algunas de las causas posibles identificadas que originan la problemática son las siguientes: a) el tiempo actual de procesamiento por unidad es considerablemente alto, esto genera que cada pedido ocupe la capacidad de la planta por un tiempo muy alto y las órdenes que lleguen deban esperar la disponibilidad del procesador; b) existe una capacidad limitada de producción debido al número insuficiente de procesadores, generando tiempos de espera obligatorios para cada orden; c) la capacidad del equipo de transporte está siendo utilizada casi en su totalidad, por lo tanto, en algunos momentos no hay vehículos disponibles en la empresa para transportar los pedidos; d) los mantenimientos preventivos se están realizando con una frecuencia incorrecta y requieren tiempos muy altos que retrasan las órdenes. Además, no se están realizando de forma correcta, propiciando fallos constantes en el sistema; e) el tiempo de cargue y descargue por pedido es muy alto, retrasando la salida de los pedidos y el regreso de los vehículos a la compañía.

\section{Establecer los objetivos}

En relación al punto anterior, el propósito del estudio de simulación estará alineado con la formulación del problema y puede responder a diferentes finalidades relacionadas con el análisis del desempeño y capacidad del sistema, la comparación de diferentes configuraciones de los elementos del sistema y su mejoramiento a través de las mismas. En este sentido los objetivos propuestos son los siguientes:

\section{Objetivo general}

Reducir en un porcentaje determinado el número de órdenes retrasadas de los clientes en una zona geográfica específica, logrando establecer la capacidad de la planta necesaria para atender la demanda actual de la empresa y mejorar el desempeño general de todos los procesos de la cadena, desde la fabricación, hasta la distribución del producto.

Objetivos específicos

Determinar el número de procesadores necesario para aumentar la capacidad de la planta. Seguidamente, identificar el nivel de mejoramiento necesario en los tiempos de procesamiento para lograr la reducción esperada en el número de órdenes rechazadas. Posteriormente, determinar el número de vehículos necesarios que garanticen disponibilidad de transporte para suplir la demanda de la empresa en el momento en que se procesan los pedidos. Por último, reducir los tiempos de cargue y descargue de pedidos a través de la regulación de dichos procesos. 


\section{Definición y conceptualización del sistema a modelar}

Se ha seleccionado como sistema de interés para la simulación una empresa ubicada en la ciudad de Manizales, Colombia, dedicada a la fabricación y distribución de productos en el Departamento de Caldas, Colombia. La empresa cuenta con la capacidad para atender gran parte del mercado existente en el departamento, llegando a 10 diferentes municipios desde su lugar de origen en la ciudad de Manizales: Aguadas, Anserma, Chinchiná, Filadelfia, La Dorada, Manzanares, Neira, Pensilvania, Riosucio y Salamina. Las características del sistema hacen que sea clasificado como un sistema discreto.

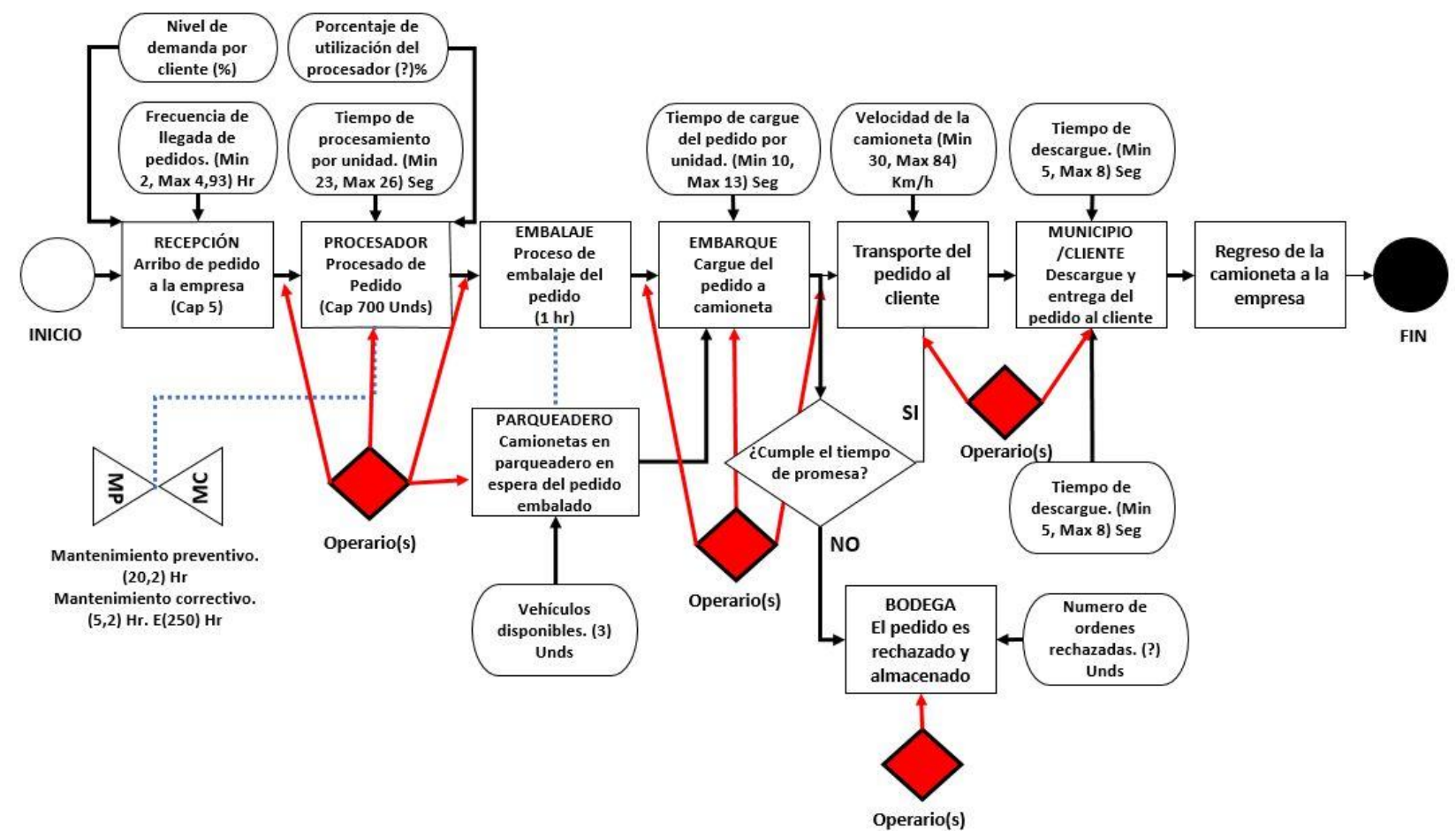

Fig. 1: Modelo conceptual proceso de fabricación y distribución realizado en Microsoft Visio basados en Educator Workshop: Teaching Simulation Using Flexim 2011 (Beaverstock y Greenwood, 2011)

Según la Fig. 1, el proceso en la empresa inicia mediante el evento originado por un cliente quien realiza la compra de un número específico de unidades. La orden de compra generada es trasladada a la fábrica en función del momento de llegada, según la regla "primero en entrar, primero en salir" (en inglés first in, first out o FIFO). El proceso de fabricación inicia en el momento en el cual la orden es recibida en la fábrica, en caso de que se esté procesando una orden, la orden nueva debe esperar la disponibilidad de la línea de producción. Debido a las características del producto, algunos clientes requieren mayores cantidades y, por lo tanto, la proporción de pedidos realizado por cada uno, así como la cantidad asociada a cada pedido, es variable y aunque están definidas por defecto en el modelo, éstas pueden ser modificadas según criterios propios del usuario. Cada pedido es procesado de forma independiente y el total de las unidades de cada orden es embalado como una sola unidad que será finalmente enviada al cliente. La empresa adopta un tiempo de promesa para cada orden requerida en el momento en que se realiza la solicitud, en caso de que el tiempo de procesamiento de la orden supere el tiempo de promesa, se asume que la orden es rechazada por el cliente y las unidades procesadas serán enviadas a una bodega como inventario disponible para suplir una nueva orden. En el momento en que se completa la orden se solicita un vehículo de la compañía para realizar la entrega de los productos. El tiempo de cargue y de descargue de la mercancía depende del número de unidades que compongan el pedido. De esta manera el tiempo de cargue es generalmente más alto que el tiempo de descargue. Cuando se termina el descargue en el lugar de destino la camioneta regresará de forma inmediata al parqueadero de la compañía y estará disponible para realizar un nuevo viaje.

\section{Recolección de datos}

Debido a la dificultad que representa realizar un análisis estadístico profundo que tenga como resultado una representación totalmente confiable del comportamiento de las diferentes variables que afectan el sistema, se ha adoptado la estimación como mecanismo base para determinar la distribución teórica de probabilidad y los valores iniciales para poner en marcha el modelo. Un ejemplo claro son las distancias existentes entre el lugar de origen y la localización de cada uno de los clientes, los cuales fueron obtenidos a través de la 
herramienta Google Maps y relacionados directamente con la herramienta a través del trazado de redes sobre un mapa a escala utilizado como fondo para la aplicación, según se ilustra en la Fig. 2.

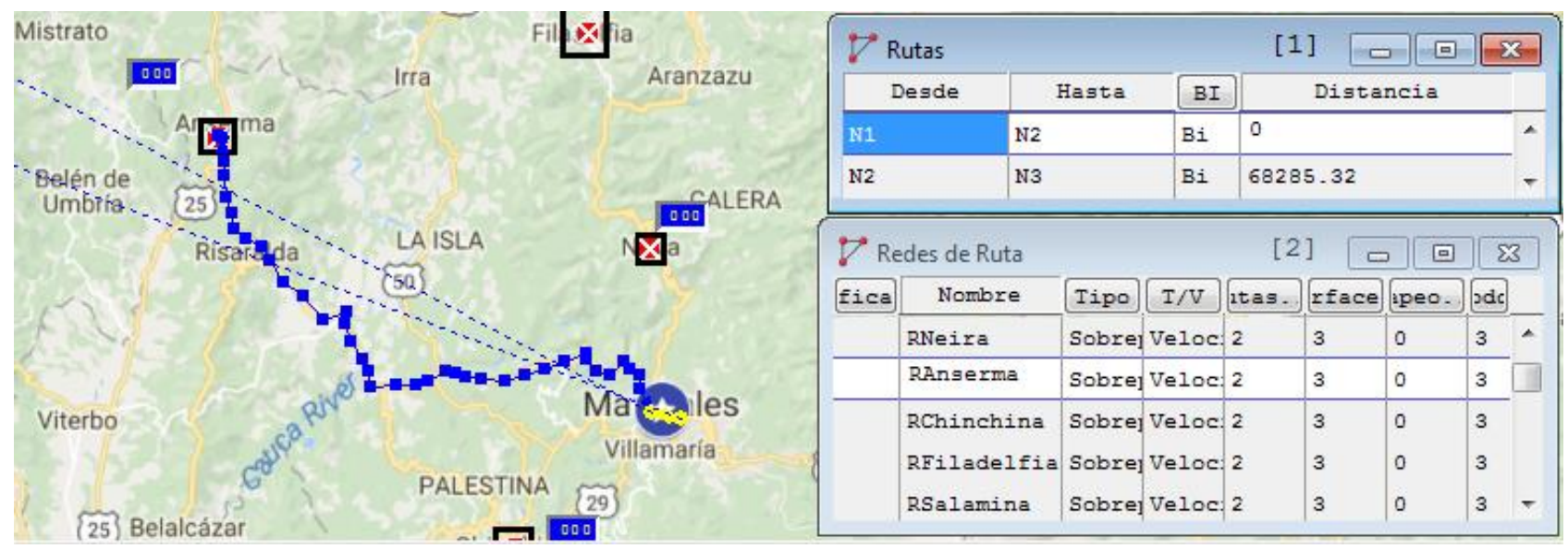

Fig. 2: Trazado de redes de ruta sobre mapa a escala en el software de simulación.

Cada uno de los valores de entrada necesarios para experimentar con el modelo (ver Fig. 3) se establecieron de forma inicial en función de las estimaciones encontradas, sin embargo, para hacer del modelo una herramienta práctica, se ha desarrollado una interfaz en Microsoft Excel que permite modificar cada uno de los parámetros a criterio de cualquier usuario que no tenga conocimiento avanzado en el uso de herramientas de simulación.
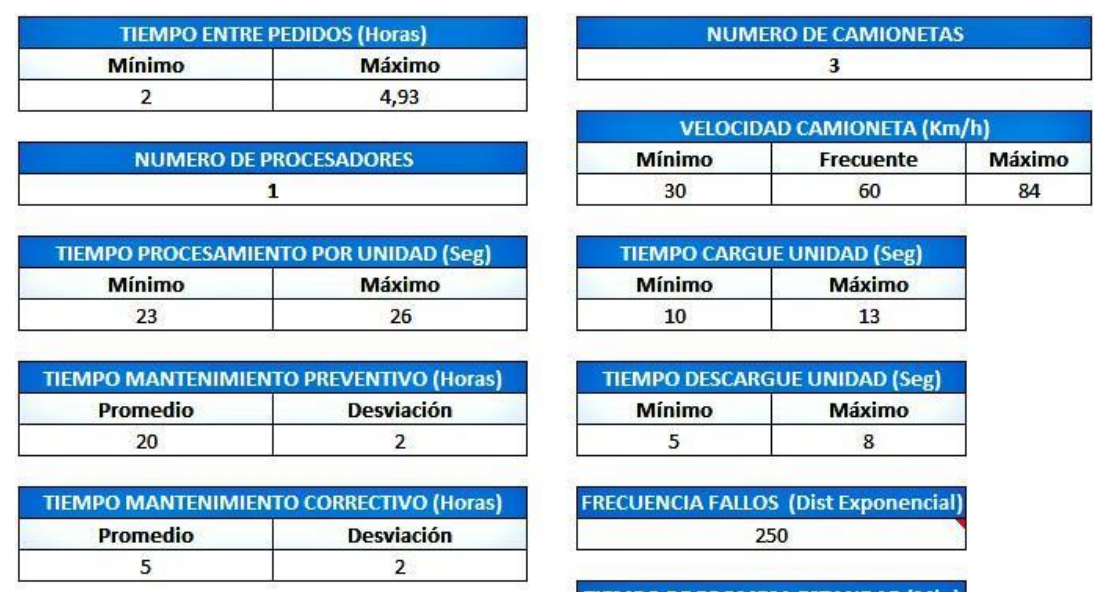

\begin{tabular}{|c|c|c|c|}
\hline \multicolumn{4}{|c|}{ REGISTRO DE CUENTES } \\
\hline \multirow{2}{*}{ Cliente } & Probabilidad & \multicolumn{2}{c|}{ Nivel de demanda } \\
\cline { 3 - 4 } & de hacer & Mínima & Máxima \\
\hline Filadelfia & 4 & 300 & 400 \\
\hline Neira & 5 & 300 & 450 \\
\hline Anserma & 5 & 300 & 500 \\
\hline Aguadas & 9 & 300 & 450 \\
\hline Pensilvania & 9 & 300 & 475 \\
\hline Riosucio & 10 & 300 & 520 \\
\hline Manzanares & 12 & 300 & 550 \\
\hline Salamina & 12 & 300 & 630 \\
\hline La Dorada & 16 & 300 & 660 \\
\hline Chinchiná & 18 & 300 & 700 \\
\hline TOTAL & 100 & & \\
\hline
\end{tabular}
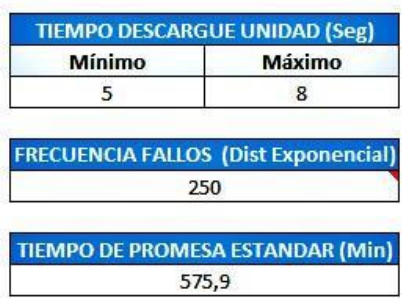

Fig. 3: Interfaz gráfica de interacción con ProModel diseñada en Microsoft Excel para modificar los niveles de las variables según los modelos de experimentación.

\section{Codificación del modelo}

Para traducir el modelo definido y conceptualizado en el modelo informático necesario para la experimentación se utilizó el software ProModel, paquete de simulación que, aunque facilita el diseño y el desarrollo del modelo a través de una interfaz gráfica, requiere la implementación de comandos específicos diseñados para la aplicación. En especial, los comandos de código son empleados durante el enrutamiento y la definición de las fases del proceso que deben seguir todos los elementos de entrada según el flujo establecido en el sistema, según se ilustra en la Fig. 4.

\section{Verificación del modelo}

Para comprobar que el modelo desarrollado incluya todos los elementos propuestos y que su funcionamiento se ajustará al diseño previsto se emplearon varios mecanismos. En primer lugar desde el inicio del proyecto se trabajó con rigurosidad para realizar un refinamiento del modelo pasa a paso. Como premisa, la construcción se llevó a cabo siguiendo las fases del proceso establecidas de forma preliminar, la recepción de la orden, su procesamiento, embalaje y transporte. 
Tabla 1: Datos del sistema y modelo para validación.

\begin{tabular}{|l|c|c|}
\hline \multicolumn{1}{|c|}{ RUTA } & SISTEMA $(\mathrm{min})$ & MODELO (min) \\
\hline Manizales-Aguadas & 133 & 109,43 \\
\hline Manizales-Anserma & 95 & 60,71 \\
\hline Manizales-Chinchiná & 36 & 27,82 \\
\hline Manizales-Dorada & 311 & 207,09 \\
\hline Manizales-Filadelfia & 62 & 43,73 \\
\hline Manizales-Manzanares & 107 & 105,73 \\
\hline Manizales-Neira & 22 & 20,23 \\
\hline Manizales-Pensilvania & 172 & 140,17 \\
\hline Manizales-Riosucio & 129 & 111,2 \\
\hline Manizales-Salamina & 109 & 73,71 \\
\hline
\end{tabular}

Mediante la "Prueba F" de Fisher es necesario la formulación de dos hipótesis: Hipótesis nula e hipótesis alterna. Estas serán validadas o no de acuerdo a los resultados generados por la prueba F. De esta manera se presentan las siguientes hipótesis.

Hipótesis nula $\left(\mathrm{H}_{0}\right)$ : $\quad$ La varianza entre los datos del sistema y los datos del modelo es igual.

Hipótesis alterna $\left(\mathrm{H}_{1}\right)$ : $\quad$ La varianza entre los datos del sistema y los datos del modelo es diferente.

Para la validación de las hipótesis planteadas con la prueba $\mathrm{F}$ se ha utilizado la herramienta de Microsoft Excel en donde al ingresar el conjunto de datos nos proporciona los valores del "F calculado" y "F crítico". Esta prueba válida o no las hipótesis si cumple las siguientes condiciones:

1. Si "F critico < F calculado"

2. Si "F critico $>$ F calculado"
Se rechaza la hipótesis nula Se rechaza la hipótesis alterna

En base a lo anterior, se ha utilizado un nivel de confianza en los datos del $95 \%$ y un alfa del $5 \%$; con el ingreso de los datos del sistema y el modelo a Microsoft Excel en la herramienta "análisis de datos" se obtienen en la tabla 2 los siguientes resultados.

Tabla 2: Resultados Prueba F.

\begin{tabular}{|c|c|c|c|c|c|c|}
\hline $\begin{array}{c}\text { Origen de las } \\
\text { variaciones }\end{array}$ & $\begin{array}{c}\text { Suma de } \\
\text { cuadrados }\end{array}$ & $\begin{array}{c}\text { Grados de } \\
\text { libertad }\end{array}$ & $\begin{array}{c}\text { Promedio de los } \\
\text { cuadrados }\end{array}$ & $\begin{array}{c}F \\
\text { calculado }\end{array}$ & $\begin{array}{c}\text { Probabilid } \\
\text { ad }\end{array}$ & $\begin{array}{c}\text { Valor crítico } \\
\text { para } F\end{array}$ \\
\hline Entre grupos & 18203,68162 & 1 & 18203,68162 & $\begin{array}{c}4,494244 \\
366\end{array}$ & $\begin{array}{c}0,048165 \\
876\end{array}$ & 4,413873419 \\
\hline $\begin{array}{c}\text { Dentro de los } \\
\text { grupos }\end{array}$ & 72907,97796 & 18 & 4050,44322 & & & \\
\hline Total & 91111,65958 & 19 & \multicolumn{5}{|l}{} \\
\hline
\end{tabular}

De acuerdo a los resultados presentados en la tabla 2 se detalla que el " $F$ crítico" es menor que el " $F$ calculado", cumpliendo la primera condición y rechazando la hipótesis nula. De igual forma, se puede comprobar la prueba $\mathrm{F}$ usando el valor de la probabilidad y el alfa, en donde si la probabilidad es menor al alfa se acepta la hipótesis alterna (caso presente). El aceptar la hipótesis alterna muestra que la varianza entre los datos del sistema y los del modelo son diferentes pero que de igual forma muestran una cercanía a la realidad.

\section{Diseño experimental}

Al ser el modelo desarrollado una herramienta basada en la metodología de enseñanza aprendizaje ABP diseñada especialmente para la experimentación, las alternativas o escenarios a ser simulados son ilimitados y por lo tanto dependen específicamente de la problemática o caso de estudio propuesta. Debe tenerse en cuenta que modificar los niveles de una o más variables tiene como consecuencia la generación de un nuevo escenario, cada uno de los cuales responde a un modelo de decisión que representa el efecto sobre las medidas de desempeño del sistema que tienen dichas modificaciones. La expresión (1) indica el modelo lógico-matemático representado a través de la simulación. En este los niveles de las $m$ variables de respuesta o medidas de desempeño $\left(y_{j}\right)$ dependen de las relaciones lógicas de las $n_{i}$ variables de decisión $\left(X_{i, j}\right)$ descritas por la función $\mathrm{f}_{\mathrm{j}}\left(\mathrm{X}_{\mathrm{i}, \mathrm{j}}\right)$ de la ec. (1). 


$$
y_{j}=f_{j}\left(x_{i, j}\right) \text { donde: } 1 \leq i \leq n_{j} ; 1 \leq j \leq m
$$

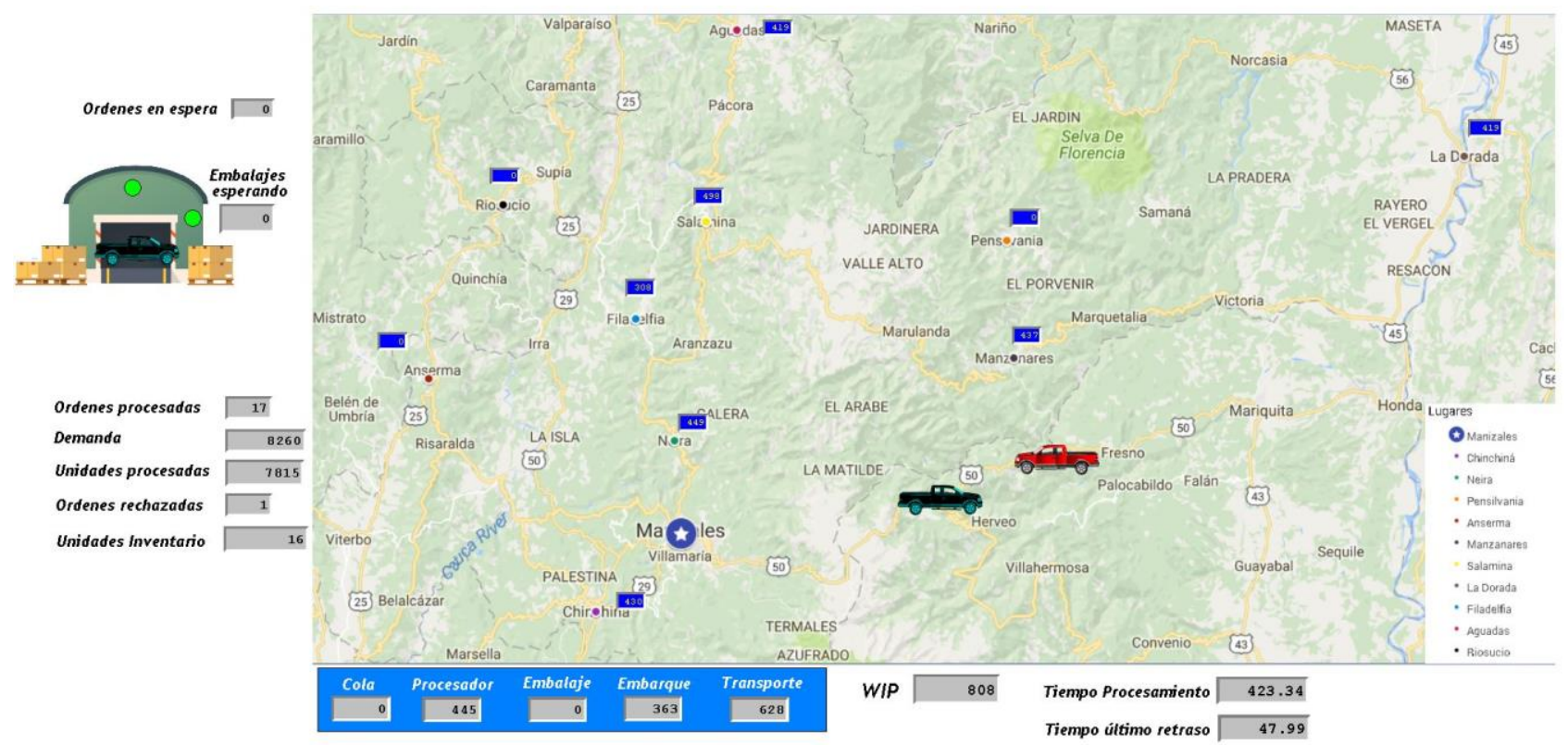

Fig. 6: Representación gráfica del modelo de simulación desarrollado en ProModel.

En la Tabla 3 se presentan posibles instancias del modelo de decisión enunciado en la expresión (1), las cuales son de aplicación práctica en el análisis de desempeño de un sistema de distribución logístico. Atendiendo al modelo de decisión, se han establecido parámetros iniciales de corrida del modelo, que a través de las estimaciones realizadas se ajustan a la situación problemática encontrada (ver tabla 4). Estos parámetros propuestos fueron sujetos a evaluación y análisis antes de ser modificados a través de la interfaz de Microsoft Excel que se provee junto con el modelo desarrollado y con ello evaluar diferentes escenarios que dieran respuesta a los objetivos planteados para la simulación. Los escenarios que satisfacen las condiciones propuestas son variados, sin embargo, atendiendo a las características del documento y a la practicidad del desarrollo, se plantean entonces dos escenarios alternativos alterando los niveles de las variables que se consideraron oportunas para atender a los objetivos propuestos.

Tabla 3: Posibles instancias del modelo de decisión.

\begin{tabular}{|c|c|c|}
\hline$X i j$ & Descripción de la variable de decisión & Medida de desempeño \\
\hline$X_{1,1}$ & Frecuencia de llegada de pedidos & \multirow{4}{*}{$\begin{array}{c}\mathrm{Y}_{1} \\
\text { Porcentaje de utilización procesador }\end{array}$} \\
\hline$X_{2,1}$ & Nivel de demanda por cliente & \\
\hline$X_{3,1}$ & Tiempo de procesamiento por unidad & \\
\hline $\mathrm{X}_{4,1}$ & Número de procesadores en el sistema & \\
\hline$X_{1,2}$ & Tiempo estándar de promesa & \multirow{7}{*}{$\begin{array}{c}\mathrm{Y}_{2} \\
\text { Número de órdenes retrasadas }\end{array}$} \\
\hline$X_{2,2}$ & Tiempo de procesamiento por unidad & \\
\hline$X_{3,2}$ & Número de vehículos disponibles & \\
\hline$X_{4,2}$ & Frecuencia de llegada de pedidos & \\
\hline$X_{5,2}$ & Frecuencia de fallos en el sistema & \\
\hline$X_{6,2}$ & Tiempo necesario mantenimiento correctivo & \\
\hline$X_{7,2}$ & Tiempo necesario mantenimiento preventivo & \\
\hline$X_{1,3}$ & Número de vehículos disponibles & \multirow{5}{*}{$\begin{array}{c}\mathrm{Y}_{3} \\
\text { Tiempo promedio de orden en el sistema por cliente }\end{array}$} \\
\hline$X_{2,3}$ & Velocidad de los vehículos & \\
\hline$X_{3,3}$ & Tiempo de procesamiento por unidad & \\
\hline$X_{4,3}$ & Tiempo de cargue & \\
\hline$X_{5,3}$ & Tiempo de descargue & \\
\hline
\end{tabular}


Tabla 4: Niveles de las variables de decisión para cada uno de los escenarios estudiados

\begin{tabular}{|l|c|c|c|}
\hline \multicolumn{1}{|c|}{ Variable de decisión } & Escenario original & Escenario 1 & Escenario 2 \\
\hline Número de procesadores & 1 & $\uparrow 2$ & $\rightarrow 1$ \\
\hline Tiempo mínimo de procesamiento por unidad (Seg) & 23 & 23 & $\downarrow 20$ \\
\hline Tiempo máximo de procesamiento por unidad (Seg) & 26 & 26 & $\downarrow 23$ \\
\hline Número de camionetas disponibles & 3 & 3 & $\uparrow 4$ \\
\hline Tiempo mínimo de cargue (Seg) & 10 & 10 & $\downarrow 6$ \\
\hline Tiempo máximo de cargue (Seg) & 13 & 13 & $\downarrow 7,8$ \\
\hline Tiempo mínimo descargue (Seg) & 5 & 5 & $\downarrow 3$ \\
\hline Tiempo máximo descargue (Seg) & 8 & 8 & $\downarrow 4,8$ \\
\hline
\end{tabular}

Análisis de salida

La fase final de la metodología adoptada se enmarca en el análisis de los resultados obtenidos mediante la experimentación al implementar diferentes configuraciones del modelo desarrollado. Estos resultados están asociados a las medidas de desempeño del sistema y su fin último es lograr que den respuesta a los objetivos planteados para la simulación. De acuerdo a lo anterior se presenta a continuación un resumen con algunas medidas de desempeño claves para el caso analizado generadas para cada uno de los escenarios planteados de forma previa.

Tabla 5: Resumen de resultados de la simulación extraídos de la plataforma Output Viewer ofrecida por ProModel

\begin{tabular}{|l|c|c|c|}
\hline \multicolumn{1}{|c|}{ Medida de desempeño del sistema } & Escenario original & Escenario 1 & Escenario 2 \\
\hline Órdenes procesadas & 202 & $\uparrow 207$ & $\uparrow 203$ \\
\hline Tiempo de espera promedio de la orden & 9,48 & $\downarrow 6,77$ & $\downarrow 1,5$ \\
\hline Tiempo promedio de una orden en el sistema & 463,24 & $\downarrow 444,57$ & $\downarrow 403,1$ \\
\hline Porcentaje de utilización procesador (Px) & 76,03 & $\downarrow \mathrm{P} 1-62,16$ & $\downarrow 74,31$ \\
\hline Número de órdenes rechazadas & 22 & $\downarrow \mathrm{P} 2-26,18$ & $\downarrow 11$ \\
\hline
\end{tabular}

\section{Análisis escenario 1}

En el primer escenario propuesto se realizó únicamente la modificación del número de procesadores del sistema, entendido como las líneas de producción con las que cuenta la empresa. Se obtuvieron resultados experimentando con dos procesadores y se logró una reducción total del número de órdenes rechazadas alcanzando el objetivo propuesto para la simulación. Si bien esta configuración es satisfactoria, debe considerarse que realizar el montaje de una línea de producción adicional implica la inversión de una cantidad de recursos significativos y por lo tanto no será siempre una opción viable para solucionar la problemática. Además, como se observa en la tabla 5 , al mantenerse los niveles de demanda constante, el número de órdenes procesadas no se incrementará de forma importante y por lo tanto se crea una capacidad ociosa en el sistema, observada en el porcentaje de utilización de ambos procesadores de $62 \%$ y $27 \%$, la cual de no ser aprovechada genera costos fijos para la organización. Por otro lado, se observa que el número de procesadores no está siendo un factor determinante en el tiempo que permanece una orden en el sistema, ya que la reducción de tiempo es únicamente del $4 \%$.

\section{Análisis escenario 2}

Atendiendo a lo anterior se considera oportuno evaluar otros escenarios modificando parámetros diferentes al número de procesadores del sistema. Para ello en un segundo escenario se han propuesto varios cambios. En primer lugar, se busca la reducción de los tiempos de procesamiento a través de la implementación de estrategias de optimización. Por otra parte, la contratación de un vehículo adicional para satisfacer los procesos de distribución junto con un auxiliar para los procesos de cargue, con lo cual se aumenta la disponibilidad de vehículos y se reducen de forma considerable los tiempos de cargue de las órdenes en la empresa. Realizar estas modificaciones en el sistema, al igual que el escenario propuesto anteriormente, es una configuración satisfactoria. Sin embargo, al realizar un comparativo de las implicaciones operacionales que tendría la implementación de cada una de las opciones, resulta viable adoptar la segunda opción 
propuesta. Se logró una reducción en las órdenes rechazadas de un $50 \%$, con lo cual se alcanza y sobrepasa el objetivo propuesto. Por otro lado, fue posible reducir en un $13 \%$ el tiempo de permanencia de una orden en el sistema, en donde se evidencia que esta medida de desempeño presenta mayor dependencia del número de vehículos con los cuales cuenta actualmente la compañía para realizar los procesos de distribución. Por último, teniendo en cuenta que los niveles de demanda y la frecuencia de llegada de pedidos se mantienen constantes, mejorar los tiempos de procesamiento tiene como consecuencia una atención más rápida de las órdenes, aunque la utilización del procesador se mantiene estable.

\section{CONCLUSIONES}

Teniendo en cuenta en que el enfoque adoptado se orienta hacia el desarrollo de una herramienta de experimentación que soporte estrategias de enseñanza-aprendizaje en el entrenamiento industrial basada en la metodología $A B P$ y que el carácter del modelado y la simulación no permite hacer una abstracción exacta del sistema real, se pueden obtener las siguientes conclusiones, se ha logrado desarrollar un modelo válido y funcional que refleja los atributos principales de un sistema logístico de distribución y permite a los interesados comprender la dinámica de los diferentes actores del sistema, así como el efecto que tiene sobre el mismo la modificación de ciertos parámetros y variables involucrados en el desempeño de este.

La característica principal de la metodología ABP es permitirles al estudiante y al profesional un acercamiento más real y completo del área a trabajar y generar una mayor comprensión del sistema. Además, gracias al diseño de la interfaz a través de Microsoft Excel, se ha dotado a la herramienta de un carácter práctico y versátil que permite a cualquier interesado con un conocimiento mínimo en software de simulación realizar la experimentación y evaluación de escenarios completamente diferenciados.

En este sentido, los resultados que pueden ser obtenidos a través de la simulación son amplios y permiten evaluar diferentes medidas de desempeño del sistema analizado y como se evidenció a través de la uso de la plataforma, fue posible realizar la comprobación de diferentes configuraciones de los parámetros del sistema, así como confrontar los efectos de diferentes decisiones que no siempre resultan ser las de mayor conveniencia y que de no ser evaluadas podrían generar efectos secundarios no deseados en el sistema o inclusive alterar negativamente el desempeño del mismo. Por último, queda en manos de los usuarios el diseño experimental que permita realizar una aplicación amplia de la herramienta en el entorno del entrenamiento industrial.

\section{REFERENCIAS}

Alles, M.A., Desarrollo del talento humano basado en competencias, $3^{\underline{a}}$ Ed., Ediciones Granica S.A, Buenos Aires, Argentina (2007)

Beaverstock, M., A. Greenwood, E. Lavery y otro autor, Applied simulation: modeling and analysis using FlexSim, $3^{a}$ Ed., FlexSim Software Products, Inc., USA (2012)

Blanco, A.C., Nuevos Análisis para la Dinámica Empresarial: Empresa, Innovación y Desarrollo, Cuadernos de Economía, 29(53), 341-348, (2010)

Bodmer, C., A. Leu, L. Mira y otro autor, Successful practices in International Engineering Education, SPINE final report, Benchmarking Study, Initial partners: Engineers Shape our Future (INGCH) and Rat der Eidgenossischen Technischen Hochschulen (ETH-Rat), Zurich, Switzerland (2002)

Caicedo, A.J., J.V. Niño y Y.A. Romero, Realidad de la capacitación y sus necesidades en los gerentes de producción del sector del calzado, cuero y sus manufacturas del Área Metropolitana de Cúcuta (Colombia), AD-minister, (23), 151-166 (2013)

Chiavenato, I., Gestión del Talento humano; un nuevo papel de los recursos humanos en las organizaciones, $1^{\text {a }}$ Ed., McGraw Hill, Bogotá, Colombia (2002)

Chiavenato, I., Gestión del Talento humano, 3ª Ed., B McGraw Hill, Bogotá, Colombia (2009)

Duque, M., Ciencia e ingeniería en la formación de ingenieros para el siglo XXI: fundamentos, estrategias y casos, ACOFI, Asociación Colombiana de Facultades de Ingeniería (2008)

Garzón, M.A. y A.L. Fisher, Modelo teórico de aprendizaje organizacional, Pensamiento \& Gestión, (24), 195-224 (2008)

Giraldo, J. A., Simulación de sistemas de producción y de servicios, 1ª Ed., Editorial Blanecolor, Manizales, Colombia (2014)

Koekemoer, E., An explorative study on factors influencing the career success of management employees, doi: 10.4102/sajip.v40i2.1204, SA Journal of Industrial Psychology, 40(2), 1-10 (2014)

Law, A.M. y W. D. Kelton, Simulation Modeling and Analysis, $3^{a}$ Ed., McGraw-Hill (2007)

López, G., C.A. Bolio y M.D. Gil, Aprendizaje organizacional: caso una panificadora, ISSN: 1815-5936, Ingeniería Industrial, 38(2), 161-170, (2017) 
Marwala, T., Semi-bounded Rationality: A model for decision making, arXiv Prepr, arXiv1305.6037 (2013-A)

Marwala, T., Flexibly-bounded Rationality and Marginalization of Irrationality Theories for Decision Making, arXiv Prepr, arXiv1306.2025 (2013-B)

Monti, L.M., N. Del' Angelo, A.M. Fernandes y otros cuatro autores, Computer and laboratory simulation in the teaching of neonatal nursing: innovation and impact on learning, Revista Latino-Americana de Enfermagem (2016)

Motsoeneng, D., A. Bezuidenhout y C. Schultz, Validation of training priorities of engineers in the platinum mining industry in South Africa, doi: 10.7166/26-3-1163, South African Journal of Industrial Engineering, 26(3), 1-11 (2015)

Norman, G.R. y H.G Schmidt, The psychological basis of problem-based learning: A review of the evidence, Academic medicine, 67(9), 557-65 (1992)

Parra, E., Mediadores cognitivos en educación superior investigación de didácticas para el pensamiento crítico, $1^{\text {a }}$ Ed., Universidad de Cartagena, Cartagena de Indias, Colombia (2006)

Rakhudu, M.A., M. Davhana-Maselesele y U. Useh, A model of collaboration for the implementation of problem-based learning in nursing education in South Africa, ISSN: 2223-6279, Curationis, 40(1), 1-10 (2017)

Seat, E. y S.M. Lord, Enabling effective engineering teams: A program for teaching interaction skills, The Research Journal for Engineering Education, 88(4), 385-390 (1999)

Sheppard, S., A. Colby, K. Macatangay y otro autor, Educating engineers: designing for the future of the field, Carnegie Foundation for the Advancement of Teaching, California, USA, (2008)

Soca, E.B., El trabajo independiente en el proceso de enseñanza-aprendizaje, ISSN: 1684-1859, Revista Cubana de Informática Médica, 7(2), 122-131 (2015)

Stable, Y., Modelo de aprendizaje organizacional para organizaciones de información, Revista Cubana de Información en Ciencias de la Salud, 22(3), 237-250 (2011)

Tortorella, G. y P.A. Cauchick-Miguel, An initiative for integrating problem-based learning into a lean manufacturing course of an industrial engineering graduate program, https://dx.doi.org/10.1590/0103-6513.224716, Production, 27, (2017)

Valderrama, M.A. y G.A. Castaño, Solucionando dificultades en el aula: una estrategia usando el aprendizaje basado en problemas, https://dx.doi.org/10.15649/cuidarte.v8i3.456, Revista Cuidarte, 8(3), 1907-1918 (2017)

Vanyolos, E., I. Furka, I. Miko y otros tres autores, How does practice improve the skills of medical students during consecutive training courses? doi; https://dx.doi.org/10.1590/s0102-865020170060000010. Rev. Acta Cirurgica Brasileira, 32(6), 491-502 (2017)

Vargas, J.M y J.A. Giraldo, Modelo de entrenamiento en toma de decisiones relacionadas con gestión de producción y operaciones de un sistema de fabricación de bioetanol, Iteckne, 12(1), 7-16 (2015)

Vazquez, G. y A. Guillamet, El entrenamiento basado en la simulación como innovación imprescindible en la formación médica, Educación Médica, 12(3), 149-155 (2009)

Villegas, J., Simulation Supported Industrial Training from an Organizational Learning Perspective, Tesis de Doctorado, Linköping University, Department of Computer and Information Science, Linköping, Sweden (1996) 
